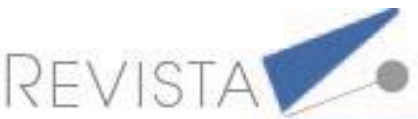

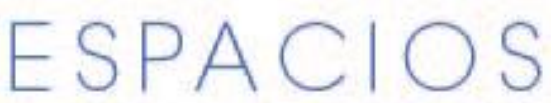

\section{La Responsabilidad Social Empresarial en las Pymes de Colombia: un estudio descriptivo a partir de las dimensiones económica, ético-legal y filantrópica ${ }^{1}$}

\section{Corporate Social Responsibility in SMEs in Colombia: a descriptive study from the economic, ethical-legal and philanthropic dimensions}

\begin{abstract}
AZUERO-RODRIGUEZ, Andrés R. ${ }^{2}$
GARCÍA-SOLARTE, Mónica ${ }^{3}$

CASTRO-RUIZ, Camilo A. ${ }^{4}$

\section{Resumen}

Los estudios de RSE en Pymes son un tema de relevancia en la actualidad dado que estas tienen un impacto en la sociedad similar o mayor al de las multinacionales. Este estudio pretende identificar la percepción de la RSE en las pymes de Colombia desde las dimensiones planteadas por Carroll: económica, ética-legal y filantrópica. Esta investigación arroja como resultado principal que los gerentes de las Pymes en Colombia dan mayor importancia en la dimensión económica de la RSE.

Palabras clave: Responsabilidad Social Empresarial, Pequeñas y Medianas Empresas, Modelo de Carroll.
\end{abstract}

\begin{abstract}
The studies of CSR in SMEs is a topic of relevance today given that they have an impact on society similar or even greater than multinationals. This study aims to identify the perception of CSR in SMEs in Colombia from the dimensions proposed by Carroll: economic, ethical-legal and philanthropic. This research shows as a main result that managers of SMEs in Colombia give greater importance to the economic dimension of CSR.

Key words: Corporate Social Responsibility, Small and Medium Enterprises, Carroll Model.
\end{abstract}

\section{Introducción}

En la actualidad se evidencia en las investigaciones de Responsabilidad Social Empresarial (de ahora en adelante RSE) que los gerentes han adquirido conciencia de sus acciones y decisiones empresariales (Herrera, Larrán y Martínez-Martínez, 2013; Herrera, Larrán, Lechuga y Martínez-Martínez, 2015a, 2015b; Herrera, Larrán, Martínez y Martínez-Martínez, 2016). El concepto de RSE es, de hecho, un punto de partida en la mayoría de los estudios, y se llega, en gran parte de estos a las mismas conclusiones: no se tiene una concepción única acerca

\footnotetext{
${ }^{1}$ El estudio se enmarca en el proyecto C.I 8140 financiado por la Vicerrectoría de Investigaciones de la Universidad del Valle.

${ }^{2}$ Profesor Asistente, Departamento de Administración y Organizaciones, Universidad del Valle, Cali, Colombia, andres.azuero@correounivalle.edu.co

${ }^{3}$ Profesora Titular, Departamento Administración y Organizaciones, Universidad del Valle, Cali, Colombia. monica.garcia@correounivalle.edu.co

${ }^{4}$ Profesor Auxiliar, Sede Palmira, Universidad del Valle, Cali, Colombia camilo.castro@correounivalle.edu.co
} 
del tema. De esta manera cada investigación abarca la RSE desde un marco de referencia que va complementando la multidimensionalidad de este concepto.

Una manera de abarcar los estudios de la RSE es la consulta con los gerentes y directivos de las organizaciones empresariales sobre los comportamientos socialmente responsables (Alvarado y Schlesinger, 2008), y, por otro lado, están los estudios que teniendo como base un marco de dimensiones, indagan sobre la percepción de las mismas específicamente desde unas escalas preestablecidas por los investigadores.

Es así como esta investigación tiene como propósito identificar la percepción de la RSE en las Pymes de Colombia, abarcando las dimensiones planteadas por Carroll (1991) en su modelo, que son: económica, ética-legal y filantrópica o discrecional. Lo anterior se abarca realizando un estudio a 492 Pymes en diferentes ciudades de Colombia, financiado por la vicerrectoría de investigaciones de la Universidad del Valle. Para dar cabida a lo anterior el artículo se desarrolla, en primer lugar, abarcando el marco teórico que expone la concepción de la responsabilidad social empresarial, la relación entre ésta y las Pymes y, la dimensionalidad de la RSE desde el modelo de Carroll; en segundo lugar, se expone la estrategia metodológica, posterior se muestran los resultados y finalmente se cierra con las conclusiones.

\subsection{Marco Teórico}

En el presente apartado se desarrollan los avances teóricos relacionados con la RSE y su vinculación con las Pymes. Adicionalmente, se pretende describir el modelo propuesto por Carroll acerca de las perspectivas económica, ética, legal y filantrópica en función de la RSE en las organizaciones empresariales.

\subsubsection{Responsabilidad Social Empresarial}

De acuerdo con autores como Carroll (1999), Nieto y Fernández (2004) Crespo (2010) y D’Andreis (2013) la RSE se discute desde mediados del siglo XX, y ésta ha abarcado diferentes definiciones e implicaciones en el desarrollo de prácticas o comportamientos empresariales. Ha sido concebida como acciones filantrópicas, éticas, económicas, legales (Carroll, 1999), entre otras, y ahora ocupa un lugar en las estrategias organizacionales. Por su parte, tal como lo expone Freeman (1984) y Popa (2010), se desarrolló la teoría de los grupos de interés o Stakeholders debido a la importancia en el marco de la RSE y porque considera la "responsabilidad" como la incidencia de las prácticas o comportamientos empresariales en diversos actores, grupos o individuos.

De esa manera, entendiendo la "empresa", desde lo expuesto por Dávila (2001), como un ente social con objetivos económicos, legales, éticos, sociales, ambientales, etc., ésta debe asumir las consecuencias correspondientes a la responsabilidad adquirida por las acciones que realiza y que tienen un efecto, positivo o negativo, en sus grupos de interés (Carroll, 1999; Sen y Cowley, 2013; Park y Ghauri, 2015; Tang y Tang, 2015). Desde este punto de vista, la RSE y los grupos de interés se convierten en un marco prescriptivo para las organizaciones empresariales, puesto que busca la manera de establecer limitaciones a las acciones que se vuelcan por incidencias negativas.

En esa misma línea argumentativa, Friedman (1970) había planteado que la responsabilidad de la empresa es hacia los accionistas, mencionando que los objetivos y acciones de las organizaciones empresariales están vinculados de manera directa con el aumento de ganancias para este grupo particular; de forma resumida, el propósito principal de todo gerente es la rentabilidad. A dicha concepción se le sumaron otras posturas acerca de las prácticas empresariales y sus repercusiones, tanto en el plano ético (Donaldson y Dunfee, 1999; Vitell y

Paolillo 2004; Brand, 2009; Özmen, 2010; Looser y Wehrmeyer, 2016) como en el contexto social, económico, ambiental y legal en los cuales inciden las acciones emprendidas por estas (Carroll, 1991, 1999; Crespo, 2010).

Se debe señalar también que, la concepción de la RSE es incidida por los avances en las TIC y la Globalización, y a raíz de lo anterior, se ha incrementado la toma de conciencia de los grupos de interés respecto a las acciones 
empresariales (Malaquias, Malaquias y Hwang, 2016; Berger-Walliser y Scott, 2018). Lo anterior deja el camino abierto a la interpretación de la RSE por parte de los gerentes, que ven la oportunidad para garantizar una imagen positiva (reputación corporativa), que finalmente va a ser incluido en la gestión y objetivos empresariales (Drucker, 1984; Dentchev, 2004; Fraj-Andrés, López-Pérez, Melero-Polo y Vázquez-Carrasco, 2012).

Mas adelante, la RSE se relaciona con investigaciones empíricas que cobijan como unidad de análisis las organizaciones empresariales, las cuales entran a exponer la falta de unificación conceptual y la exposición de las diversas acciones que estas desarrollan en función de los grupos de interés y, en pro de sus objetivos (Crespo, 2010). Por ejemplo, la relación de las acciones de RSE con el "desarrollo sostenible", concepto que se comienza a tratar internacionalmente a inicios del siglo XXI, con la propuesta de "Los Objetivos del Milenio" (Dubois, 2006), y que actualmente se nombra como "Objetivos de Desarrollo Sostenible -ODS" (Coronado, 2016). Estos últimos se establecen en la Cumbre de Desarrollo Sostenible realizada en el 2015 por los Estados miembro de la ONU y otros actores relevantes. De manera resumida, estos involucran las acciones de diferentes actores en la sociedad, entre los cuales se encuentran las organizaciones empresariales como generadoras de progreso y desarrollo (económico, social y ambiental), y los Estados Nación y sus instituciones representantes.

Teniendo en cuenta lo anterior, la RSE se vuelve relevante y se considera un tema en desarrollo en el contexto académico y empresarial, incluso para al planteamiento de políticas públicas relacionadas con los ODS. Al respecto del ámbito académico, se busca describir y explicar los fenómenos suscitados en las organizaciones empresariales, para lo cual existen estudios teóricos, en los cuales la RSE se clasifica, se explica, se interpreta, se comprende, y busca indagar acerca de los actores ante los cuales estas deben responder (Wang, Tong, Takeuchi y George, 2016).

En cuanto al contexto empresarial, los gerentes/propietarios buscan la comprensión del concepto, su desarrollo, la relación con la gestión y las implicaciones en diferentes variables organizacionales (Crespo, 2010). Un ejemplo relacionado es la clasificación de las prácticas de RSE expuestas por Hamburger (2007) y Miranda y Cantallops (2013) en las que definen por niveles o jerarquías las acciones ejecutadas por las organizaciones empresariales.

\subsubsection{RSE y Pymes}

Atendiendo las consideraciones anteriores, se prosigue de manera particular en la relación entre la RSE y las Pymes, aclarando que el predominio de los estudios se ha enmarcado de manera extensa en las grandes empresas. Es importante resaltar que las Pymes son consideradas motores de la economía, generadoras de riqueza (indicador ligado al PIB) y alicientes para la generación de empleo (Mellado, 2009). Tal como lo plantea Vargas (2002) las Pymes son relevantes debido al impacto social, económico y ambiental. En resumen, dinamizan y generan tejido social y económico en función de redes empresariales emergentes, se disminuye el desempleo, y hay aumento en el consumo de bienes y servicios (Franco y Urbano, 2010).

Al respecto de la relación entre la RSE y las Pymes, autores como Enderle (2004), Herrera, Larrán y MartínezMartínez (2013), Herrera, Larrán, Lechuga y Martínez-Martínez (2015a, 2015b), Fraj-Andrés, et al., (2012) plantean que las acciones "responsables" son implementadas debido a la repercusión positiva (calidad de vida y bienestar, imagen o reputación corporativa, reforzamiento del fundamento ético del gerente, entre otras variables) en la percepción de los grupos de interés.

Por su parte, de acuerdo con Herrera, et al. (2015a, 2015b), es casi imperceptible la exposición de las prácticas de RSE de parte de las Pymes, esto comprado con la difusión que realizan las grandes empresas. Los autores manifiestan que las variables de estudio de las organizaciones empresariales de mayor trascendencia son la estructura, los rasgos del directivo y/o dueño (el género y la formación) y el sector. Resaltan también que el desempeño, la productividad, la competitividad, la innovación, la imagen o reputación corporativa, relaciones 
con la comunidad, entre otros resultados destacados de las organizaciones empresariales son parte de las evidencias empíricas que se resaltan de los estudios de la RSE en relación con las Pymes.

Para citar un ejemplo de lo anterior, el estudio de Preuss y Perschke (2010) evidencia que los grupos de interés a los cuales las Pymes dirigen sus estrategias y acciones desarrollan una percepción positiva, en la medida que se ven incluidos en la implementan dichas acciones. Entre los resultados que obtienen estos autores, se encuentra que los empleados y los clientes son los grupos de interés en los que se concentran la mayor cantidad de acciones y prácticas de RSE.

A manera de complemento de lo anterior, investigaciones realizadas por Aya y Sriramesh (2014), Ayuso, Roca y Colomé (2013), Battaglia, Testa, Bianchi, Iraldo y Frey (2014), Laudal (2011), Rahim y Wisuttisak (2013), exponen que los grupos de interés en los que se focalización las acciones de RSE son los clientes y proveedores. Estos últimos dan trascendencia a los principios y valores que las organizaciones empresariales trasmiten a nivel interno y externo con sus acciones, tales como la confianza, integridad, transparencia y honestidad. Lo anterior lo relacionan con las características personales del comportamiento del gerente/propietario (personalidad, educación, género, etc.) y la cultura (ligada a la idiosincrasia y la ubicación geográfica).

Se resalta también que el área funcional de las organizaciones empresariales en la cual se manifiestan más acciones de RSE en las Pymes, es la gestión humana (Enderle, 2004; Vives, Corral y Isusi, 2005; Mellado, 2009; Herrera, et al., 2015a; Coppa y Sriramesh, 2013). Las acciones, de acuerdo con los autores, están dirigidas al equilibrio entre trabajo y familia, el desarrollo profesional, la puesta en marcha del plan carrera, la seguridad, la salud, el bienestar y la calidad de la vida laboral. Los autores también exponen que los colaboradores como grupo de interés producen mejoras en el desempeño de las Pymes, por lo cual, tienden a ser la razón de la implementación del mayor número de acciones de RSE. No obstante, Ocampo-López, García-Cortés, Ciro-Ríos y Forero-Páez (2015), contradicen lo mencionado, y plantean que la explicación de que las acciones de RSE estén orientadas a los colaboradores, es el cumplimiento legal, puesto que es de carácter obligatorio.

\subsubsection{Multidimensionalidad de la RSE}

Ahora bien, para el presente estudio es necesario especificar que la RSE ha sido estudiada desde diferentes perspectivas o dimensiones (Vera y Peláez, 2013; Alvarado y Schlesinger, 2008; Pérez, García de los Salmones, Rodríguez, 2008; García de los Salmones, Herrero, Rodríguez, 2005), sin embargo, desde que aparece en el panorama académico el modelo de Carroll $(1979,1991,1999)$, la RSE y los estudios que de ésta se realizan buscan comprender las expectativas económicas, éticas, legales y filantrópicas que se tiene sobre las organizaciones empresariales. En este sentido, de acuerdo con Carroll (1999), las dimensiones para investigar la RSE son cuatro: dimensión económica, ética, legal y filantrópica, descritas en la Tabla 1, a continuación.

Tabla 1

Dimensiones de la RSE

\begin{tabular}{ll}
\hline \multicolumn{1}{c}{ Dimensión } & \multicolumn{1}{c}{ Descripción } \\
\hline \multirow{2}{*}{ Económica } & $\begin{array}{l}\text { Se espera que las organizaciones empresariales sean rentables y } \\
\text { productivas, además que obtenga utilidades, después de lograr los } \\
\text { presupuestos que se realicen de ventas de los productos o servicios que } \\
\text { ofrece. }\end{array}$ \\
\hline & $\begin{array}{l}\text { Al respecto de esta dimensión, se establece que las organizaciones } \\
\text { empresariales deben alcanzar sus objetivos sustentándose en las } \\
\text { Legal } \\
\text { obligaciones de Ley. En pocas palabras, su razón de ser se cobija bajo } \\
\text { lineamientos normativos nacionales y supranacionales, al igual que sus } \\
\text { operaciones y los medios para alcanzar sus objetivos. }\end{array}$ \\
\hline \multirow{2}{*}{ Ética } & La sociedad espera que todas las acciones y comportamientos que se \\
& derivan de las operaciones empresariales estén amparadas por normas \\
\hline
\end{tabular}




\begin{tabular}{ll}
\hline & $\begin{array}{l}\text { éticas. Es decir, el desempeño debe ampararse en valores y principios } \\
\text { morales que de la sociedad se reflejan. }\end{array}$ \\
\hline Filantrópica & $\begin{array}{l}\text { En la sociedad existen diversas problemáticas de las cuales se espera, de } \\
\text { parte de las organizaciones empresariales, se involucren para dar } \\
\text { Discrecional }\end{array}$ \\
\hline & $\begin{array}{l}\text { solución, estableciendo un rol en función del mejoramiento y progreso } \\
\text { de ésta. }\end{array}$ \\
\hline
\end{tabular}

Fuente: adaptado de Carroll (1999)

De acuerdo con Maignan y Ferrell (2003), a partir del modelo planteado por Carroll $(1991,1999)$ se puede establecer que las expectativas que tiene la sociedad al respecto del accionar de las organizaciones empresariales, en relación con las dimensiones de la RSE, es congruente con las acciones reales emprendidas por las empresas y que son reveladas por los gerentes. En relación con lo anterior, a partir de estudios realizados de las expectativas de los actores o grupos de interés acerca de las dimensiones definidas por Carroll en su modelo, se establece a partir de un Análisis Factorial de Correspondencias, que la dimensión ética y legal se constituyen como una sola (García de los Salmones, et. al, 2005). De ahí que Alvarado y Schlesinger (2008) establecieran en su análisis que el instrumento para el análisis de la RSE de las empresas estuviese categorizado por tres dimensiones: económica, ético legal y filantrópica.

\section{Metodología}

En el presente apartado se aborda la metodología empleada en la investigación, se presenta la muestra, el instrumento utilizado, las variables estudiadas y la validez de esta.

\subsection{Tipo y enfoque de investigación}

Esta es una investigación cualitativa y descriptiva que permite describir las características de las pymes en Colombia y las prácticas de RSE que emplean. Esta investigación fue realizada a través de un cuestionario autoadministrado a los gerentes de las de tres principales ciudades de Colombia como son: Pymes de Cali, Medellín y Bogotá.

\subsection{Muestra}

Para calcular la muestra se realiza un muestreo aleatorio simple con un nivel de confianza del 95\%, un margen de error del $5 \%$ y una probabilidad de éxito y fracaso del 50\%. Para realizarlo se tiene en cuenta el total de Pymes en las tres ciudades (Cali, Medellín y Bogotá). En la Tabla 2, se presenta los datos por ciudad. Los registros mercantiles son adquiridos en las Cámaras de Comercio de Cali y Bogotá (esta última contiene Bogotá y Medellín).

Tabla 2

Muestra

\begin{tabular}{cc}
\hline Ciudad & No. De Pymes \\
\hline Bogotá & 207 \\
\hline Cali & 193 \\
\hline Medellín & 92 \\
\hline Total & 492 \\
\hline Fuente: elaboración propia
\end{tabular}

Fuente: elaboración propia.

\subsection{Variables}

\subsubsection{Características demográficas de las Pymes}


A continuación, definiremos las variables demográficas con que se describen en esta investigación las pymes de Colombia.

Género: En el trabajo utilizamos el concepto de género en relación con el sexo del gerente. Siendo una variable categórica que toma el valor 1 cuando la empresa es gestionada por mujer y 0 cuando es gestionada por hombre.

Localización de la Pyme: en esta investigación se trabajó con tres de las principales ciudades de Colombia. Siendo 1. Bogotá, 2. Medellín y 3. Cali.

Sector: en esta investigación las empresas se clasificaron en tres sectores, primario que es asociado a la agricultura, la pesca y la ganadería, secundario que es asociado a la industria y terciario asociado al comercio y servicios.

Formación del gerente: se considera a partir de una variable que toma tres valores de acuerdo con el nivel máximo alcanzado por el gerente. Siendo 1. Bachiller, 2. técnico y 3. Formación Universitaria.

Carácter familiar de la empresa: se identifica a partir de una variable dicotómica que tiene valor 1 cuando la mayoría de la propiedad de la empresa se encuentra en manos de una familia, y valor 0 en contrario.

Liderado por: se identifica a partir de una variable dicotómica que tiene valor 1 cuando es liderada por alguien de la familia y 0 . Cuando es liderada por un externo.

\subsubsection{Prácticas de RSE en las Pymes}

Para medir la RSE se adaptó el instrumento de Alvarado y Schlesing (2008) que mide la RSE en tres dimensiones: económica, ético legal y filantrópica, las cuales fueron adaptadas de Alvarado y Schlesinger (2008). Los ítems de las dimensiones son construidos en una escala tipo Likert de 7 posiciones, con límites $1=$ completamente en desacuerdo a 7 = completamente de acuerdo.

Para corroborar la validez de todas las variables se llevó a cabo un análisis factorial en el que tanto las cargas factoriales como el coeficiente KMO y la prueba de esfericidad de Bartlett arrojan resultados suficientes para validar el indicado obteniéndose los siguientes resultados Económica KMO:0,500, varianza explicada 72,108\%; Etico-Legal KMO: 0,785, varianza explicada 65,174\%; y Filantrópica KMO: 0,500, varianza explicada 73,581\%. En términos de confiabilidad, se utiliza el alfa de Cronbach se obtuvieron los siguientes resultados: Económica $(0,6081)$, Ético-legal $(0,8090)$ y Filantrópica $(0,6769)$. Los resultados muestran que existe una fiabilidad aceptable en los factores. Los resultados de validación son aceptables, por lo que se decide trabajar con estas variables

\section{Resultados}

A continuación, se caracterizan las Pymes y se procede a exponer la percepción de la RSE desde la dimensión económica, ético-legal y filantrópica o discrecional, de las mismas.

\subsection{Caracterización de las Pymes}

Tal como se muestra en la Tabla 3, de la muestra de 492 Pymes, el $25 \%$ son gestionadas por mujeres y el $75 \%$ restante es gestionada por hombres. Con respecto a la distribución de estas Pymes en el país, se tiene que el $42,07 \%$ pertenecen a Bogotá, el 39,23\% en Cali y el 18,70\% restante a Medellín. Por su parte, el sector dominante es el terciario con el 66,06\% de la muestra; esto indica que el comercio y los servicios tienen predominancia entre las pequeñas y medianas empresas; el $25,41 \%$ y el $8,54 \%$ son del sector secundario o industria y primario respectivamente, este último asociado a la agricultura, la pesca y la ganadería. 
Tabla 3

Características de las Pymes de la muestra

\begin{tabular}{|c|c|c|}
\hline Característica & Frecuencia & $\%$ \\
\hline \multicolumn{3}{|c|}{ Género del Gerente } \\
\hline Hombre & 123 & $25 \%$ \\
\hline Mujer & 369 & $75 \%$ \\
\hline \multicolumn{3}{|c|}{ Localización de la Pyme } \\
\hline Bogotá & 207 & $42,07 \%$ \\
\hline Cali & 193 & $39,23 \%$ \\
\hline Medellín & 92 & $18,70 \%$ \\
\hline \multicolumn{3}{|c|}{ Sector } \\
\hline Primario & 42 & $8,54 \%$ \\
\hline Secundario & 125 & $25,41 \%$ \\
\hline Terciario & 325 & $66,06 \%$ \\
\hline \multicolumn{3}{|c|}{ Formación del Gerente } \\
\hline Bachiller & 31 & $6,30 \%$ \\
\hline Técnica & 6 & $1,22 \%$ \\
\hline Formación Universitaria & 455 & $92,48 \%$ \\
\hline \multicolumn{3}{|c|}{ Carácter Familiar } \\
\hline Carácter Familiar & 144 & $29,27 \%$ \\
\hline No Familiar & 348 & $70,73 \%$ \\
\hline Lideradas por la familia & 170 & $34,55 \%$ \\
\hline Liderada por externos & 322 & $65,45 \%$ \\
\hline
\end{tabular}

En la Tabla 3, también se muestra que entre los pequeños y medianos empresarios hay un alto número de gerentes que tienen formación profesional (92,48\%), lo cual es fundamental en la dirección de este tipo de organizaciones. Otro dato importante es que en promedio la antigüedad de las Pymes de la muestra es de 30 años, lo cual es relevante cuando se busca indagar sobre las prácticas de RSE, siendo estas más destacadas entre los gerentes de estas organizaciones empresariales.

La edad promedio de los gerentes es de 54 años y llevan aproximadamente 20 años ejerciendo el cargo. De la muestra, el $29,27 \%$ de las Pymes son de carácter familiar y el 34,55\% de las empresas exponen que los cargos directivos recaen sobre los miembros de la familia.

\subsection{Descriptivos de la RSE}

Tal como se expone en el marco teórico, la RSE se mide desde las dimensiones Económica, Ético-Legal y Filantrópica expuestas por Carroll (1991). Las dimensiones son valoradas con 8 afirmaciones de la encuesta. A continuación, en la Tabla 4, se presenta la media y desviación estándar de cada uno de estas. 
Tabla 4.

Estadísticos descriptivo

Responsabilidad Social Empresarial

\begin{tabular}{|c|c|c|c|}
\hline Variable & Ítem & Media & $\begin{array}{l}\text { Desviación } \\
\text { Estándar }\end{array}$ \\
\hline \multirow[t]{2}{*}{ Económica } & $\begin{array}{l}\text { 1. Siempre intenta obtener la máxima utilidad } \\
\text { económica de su actividad }\end{array}$ & 5,665 & 1,3324 \\
\hline & 2. Intenta obtener éxito a largo plazo & 5,74 & 1,5559 \\
\hline \multirow{4}{*}{ Ético legal } & $\begin{array}{l}\text { 3. Se preocupa de cumplir sus obligaciones con sus } \\
\text { accionistas, proveedores, distribuidores y otros } \\
\text { grupos con los que trata }\end{array}$ & 5,776 & 1,1624 \\
\hline & $\begin{array}{l}\text { 4. Se comporta ética/honestamente con sus } \\
\text { clientes }\end{array}$ & 5,14 & 1,5031 \\
\hline & $\begin{array}{l}\text { 5. Da prioridad a sus principios éticos en sus } \\
\text { relaciones en lugar de solo priorizar el desempeño } \\
\text { económico }\end{array}$ & 5,122 & 1,6388 \\
\hline & $\begin{array}{l}\text { 6. Se preocupa por respetar y proteger el medio } \\
\text { ambiente }\end{array}$ & 5,299 & 1,3547 \\
\hline \multirow{2}{*}{ Filantrópica } & $\begin{array}{l}\text { 7. Patrocina o financia activamente eventos y } \\
\text { actividades con fines sociales (deportes, música...) }\end{array}$ & 4,868 & 0,9809 \\
\hline & $\begin{array}{l}\text { 8. Destina parte de su presupuesto a donaciones y } \\
\text { trabajos sociales }\end{array}$ & 4,746 & 1,3934 \\
\hline
\end{tabular}

Fuente: Elaboración propia

Ahora bien, teniendo en cuenta la Tabla 4, en promedio, los gerentes se preocupan en mayor medida por cumplir con las expectativas que tienen de la organización empresarial a los accionistas, proveedores, distribuidores y otros grupos con los que trata. Después viene una preocupación por el éxito a largo plazo y la obtención de la máxima utilidad económica. La responsabilidad que cuenta con un menor grado de preocupación entre los gerentes es el desarrollo de patrocinios en eventos y actividades con fines de lucro, relacionados con la música, el deporte, entre otros. En términos generales, de acuerdo con la Tabla 5, la dimensión a la cual le dan mayor importancia los gerentes de las Pymes de la muestra es a la responsabilidad Económica, la cual está encaminada al desempeño económico, las utilidades y la estabilidad en el largo plazo.

Tabla 5

Responsabilidad Social Empresarial en las Pymes

\begin{tabular}{cc}
\hline Dimensión & Promedio \\
\hline Económica & 5,7022 \\
\hline Ético legal & 5,3343 \\
\hline Filantrópica & 4,8069 \\
\hline
\end{tabular}

Fuente: elaboración propia

Los resultados de la Tabla 4 y Tabla 5, exponen que pesa más la dimensión económica, mientras que la filantrópica tiene menos interés. Esto concuerda con lo planteado por Carroll (1991) en sus estudios y en las investigaciones que se han llevado a cabo bajo el mismo modelo, como la de Maignan y Ferrell (2000), Pinkston y Carroll (1996), Burton y Hegarty (1999) y Ibrahim y Angelidis (1993). Los primeros sugieren que las acciones encaminadas a las dimensiones planteadas por Carroll propenden por el desarrollo de lo que han denominado "ciudadanía corporativa". Eso implica una preocupación por cada una de las obligaciones: económicas, éticas, legales y discrecionales, que tienen los gerentes de las organizaciones empresariales. Por su parte, para los 
segundos, realizan un estudio empírico en el cual evidencian que las prioridades de los gerentes de filiales químicas multinacionales con sede en EE.UU. son la responsabilidad económica, seguida por las legales.

A partir de lo anterior en la Tabla 6 se analizan estos componentes considerando el género del gerente.

Tabla 6

Responsabilidad Social Empresarial en las

Pymes según el género del gerente

\begin{tabular}{ccc}
\hline \multirow{2}{*}{ Dimensión } & \multicolumn{2}{c}{ Género del Gerente } \\
\cline { 2 - 3 } & $\begin{array}{c}\text { Hombre } \\
\text { (prom) }\end{array}$ & $\begin{array}{c}\text { Mujer } \\
\text { (prom) }\end{array}$ \\
\hline Económica & 5,7357 & 5,6016 \\
\hline Ético Legal & 5,3462 & 5,2987 \\
\hline Filantrópica & 4,8292 & 4,7398 \\
\hline
\end{tabular}

Fuente: elaboración propia

De acuerdo con los datos de la Tabla 6, la dimensión que predomina entre los gerentes tanto hombres como mujeres es la económica, siendo para los hombres un promedio más alto que en las mujeres.

Tabla 7

Responsabilidad Social Empresarial en as Pymes según el sector de la Pymes

\begin{tabular}{cccc}
\hline & \multicolumn{3}{c}{ Sector } \\
\hline Dimensión & Primario & Secundario & Terciario \\
\hline Económica & 5,655 & 5,708 & 5,706 \\
\hline Ético Legal & 5,619 & 5,179 & 5,375 \\
\hline Filantrópico & 4,698 & 5,005 & 4,946 \\
\hline & Fuente: Elaboración propia
\end{tabular}

Al respecto de la Tabla 7, evidencia que la responsabilidad económica es la más importante para todos los sectores. En ésta el sector secundario y terciario tienen poca diferencia entre ambas y sobrepasan en una décima la percepción del sector primario. Por otro lado, la responsabilidad ética-legal es muy importante para el sector primario, superando al sector secundario y terciario por más de 2 décimas. Finalmente, para el sector secundario en comparación con los otros dos, la responsabilidad filantrópica o discrecional es más importante.

Tabla 8

Responsabilidad Social Empresarial en las

Pymes según la formación del gerente

\begin{tabular}{cccc}
\hline & \multicolumn{3}{c}{ Formación } \\
\hline Dimensión & Bachillerato & Técnico & Universitario \\
\hline Económica & 5,210 & 5,750 & 5,735 \\
\hline Ético Legal & 5,204 & 5,833 & 5,349 \\
\hline Filantrópico & 5,000 & 4,889 & 4,936 \\
\hline & Fuente: elaboración propia
\end{tabular}

De acuerdo con la Tabla 8, la responsabilidad económica y la ética-legal es percibida en mayor medida por los gerentes con formación técnica, y la responsabilidad filantrópica por los gerentes con formación de media (bachillerato). Por su parte, los gerentes con formación universitaria o profesional perciben con un promedio de 5,735 y de 5,349 la responsabilidad económica y ético-legal respectivamente, ubicándose por encima de la percepción de los gerentes con formación media y por debajo de los gerentes con formación técnica. 
Tabla 10

Responsabilidad Social Empresarial en las

Pymes según la ubicación de la Pyme

\begin{tabular}{cccc}
\hline & \multicolumn{3}{c}{ Ubicación de la Pyme } \\
\hline Dimensión & Bogotá & Medellín & Cali \\
\hline Económica & 6,019 & 5,446 & 5,484 \\
\hline Ético Legal & 5,422 & 4,569 & 5,636 \\
\hline Filantrópica & 4,919 & 5,170 & 4,851 \\
\hline
\end{tabular}

Fuente: elaboración propia

De manera general, teniendo en cuenta la ubicación geográfica de las Pymes en Colombia (Ver Tabla ), se logra constatar que la responsabilidad económica es prioridad en Bogotá, mientras que en Cali prima la preocupación ético legal en este tipo de organizaciones empresariales. De manera individual, en Bogotá, Medellín y Cali la preocupación por la maximización de las utilidades y el éxito a largo plazo de las compañías es lo más relevante. Por su parte, en Bogotá y Cali la preocupación por la responsabilidad discrecional o filantrópica es lo tercero en lo que piensan los gerentes de las pymes. En Medellín por su parte, es más importante las acciones encaminadas a la filantropía que lo normativo, ético o legal.

Tabla 9

Responsabilidad Social Empresarial en las Pymes según el carácter familiar

\begin{tabular}{ccc}
\hline & \multicolumn{2}{c}{ Composición Familiar } \\
\hline Dimensión & SI & NO \\
\hline Económica & 5,910 & 5,616 \\
\hline Ético Legal & 5,174 & 5,418 \\
\hline Filantrópico & 5,032 & 4,901 \\
\hline
\end{tabular}

Fuente: elaboración propia

En cuanto al carácter de la gerencia de las pymes, la composición familiar de las mismas tiene un peso relevante, porque hace que las tres dimensiones de la RSE, de acuerdo con el modelo de Carroll (1991) puntúan por encima del 5,0. De estas, la preocupación por las utilidades y lo económico prima, al igual que las empresas que no son de carácter familiar, sin embargo, estas últimas no consideran tan importante las acciones filantrópicas.

\section{Conclusiones}

El estudio de la percepción de la RSE desde las dimensiones planteadas por Carroll (1991), establece que las organizaciones empresariales deben mostrar interés por la dimensión económica, ético-legal y filantrópica o discrecional. Es decir, mostrar preocupación por garantizar la utilidad financiera y el éxito a largo plazo, el cumplimiento de las obligaciones adquiridas con sus grupos de interés (accionistas, proveedores, distribuidores, entre otros), la exposición de un comportamiento ético y honesto, dando prioridad a principios éticos, además por dar muestra de una preocupación por el medio ambiente. Adicionalmente, se plantea que los gerentes deben mostrar interés por patrocinar eventos o destinar un presupuesto para donaciones y trabajos de índole social.

De manera general, los gerentes de las Pymes en Colombia dan prioridad a la dimensión económica, preocupándose por la maximización de las utilidades y el éxito a largo plazo de sus organizaciones empresariales; de acuerdo con lo mencionado y al hacer el contraste con el modelo teórico planteado por Carroll, el comportamiento de las pymes coincide con los escalones la pirámide planteada por el autor, siendo el económico la preocupación de base para las empresas. Lo anterior también es acorde con los objetivos que este tipo de organizaciones tiene, siendo en cantidad, la mayoría en el país (más del $90 \%$ en Colombia) y buscando pasar el valle de la muerte, subsistiendo día a día. Lo anterior coincide con lo mencionado por Carroll (2016), "la infraestructura de RSE se basa en la premisa de que un negocio sea económicamente sólido y sostenible". 
Adicional, cabe resaltar que a pesar de ser lo económico una preocupación de base e importante para los gerentes en Colombia, la dimensión ética legal tiene una percepción alta, lo cual indica que las obligaciones de ley, tener un comportamiento honesto, dar prioridad a los principios éticos, y respetar el medio ambiente, representan su quehacer, mostrando que tienen un interés por este tipo necesidades. En cuanto a la dimensión filantrópica, se muestra que en Colombia los pequeños y medianos empresarios tienen interés por acciones voluntarias o por la participación en eventos y actividades de donación, pero no son la prioridad.

En cuanto a los resultados por dimensión y cada una de las características de las pymes se logra concluir que respecto al género del gerente tanto hombres como mujeres dan prioridad a la dimensión económica; seguido de la dimensión ético legal y se cierra con la dimensión filantrópica. Por su parte, para los tres sectores económicos, la maximización de las utilidades y las proyecciones a largo plazo es lo más importante; cabe resaltar que para el sector secundario es predominante la dimensión económica en comparación del primario y terciario; adicional, la preocupación por la realización de donaciones o actos filantrópicos resalta, en contraste con los otros dos. Por su parte, para el sector primario predomina, en comparación con los otros dos sectores, la dimensión ético legal.

En cuanto la formación del gerente se destaca que el nivel técnico da prioridad a las necesidades económicas de las pymes, aunque los otros dos niveles de formación están de acuerdo en que la dimensión económica es importante, los gerentes con formación técnica resaltan en mayor medida ésta. Se evidencia que los gerentes con formación básica media en Colombia conocido como bachillerato, dan importancia a las acciones de orden filantrópico en mayor medida que los gerentes de formación técnica y universitaria. Por su parte, la dimensión ético legal es preponderante para los gerentes de nivel técnico, siendo ésta la más importante entre los gerentes de este nivel de formación.

En cuanto a la ubicación de las pymes, en Bogotá existe una mayor preocupación por la responsabilidad económica; en Cali por su parte, la dimensión ético legal predomina, y en Medellín en comparación de las dos ciudades anteriores destaca su preocupación por actos filantrópicos. Finalmente, respecto a la composición de las pymes, es decir si es familiar o no, para aquellas pymes que son familiares es más importante la dimensión económica y filantrópica. Esto sin dejar de lado que para las empresas que no se componen por la familia también es importante la económico, y se suma la responsabilidad lo ético legal.

\section{Referencias bibliográficas}

Alvarado H., A. \& Schlesinger D., M. W. (2008). Dimensionalidad de la responsabilidad social empresarial percibida y sus efectos sobre la imagen y la reputación: una aproximación desde el modelo de Carroll. Estudios gerenciales, 24(108), 37-59.

Aya Pastrana, N., y Sriramesh, K. (2014). Corporate Social Responsibility: Perceptions and practices among SMEs in Colombia, Public Relations Review, 40(1), 14-24. http://doi.org/10.1016/j.pubrev.2013.10.002

Ayuso, S., Roca, M., y Colomé, R. (2013). SMEs as "transmitters" of CSR requirements in the supply chain. Supply Chain Management: An International Journal, 18(5), 497-508.

Battaglia, M., Testa, F., Bianchi, L., Iraldo, F., y Frey, M. (2014). Corporate social responsibility and competitiveness within SMEs of the fashion industry: Evidence from Italy and France. Sustainability, 6(2), 872-893.

Berger-Walliser, G., \& Scott, I. (2018). Redefining corporate social responsibility in an era of globalization and regulatory hardening. American Business Law Journal, 55(1), 167-218. 
Brand, V. (2009). Empirical business ethics research and paradigm analysis. Journal of business ethics, 86(4), 429-449.

Burton, B. K., \& Hegarty, W. H. (1999). Some determinants of student corporate social responsibility orientation. Business \& society, 38(2), 188-205.

Carroll, A. B. (1991). The pyramid of corporate social responsibility: Toward the moral management of organizational stakeholders. Business Horizons, 34(4), 39-48.

Carroll, A. B. (1999). Corporate Social Responsibility: Evolution of a Definitional Construct. Business and Society, $38(3), 268-295$.

Carroll, A. B. (2016). Carroll's pyramid of CSR: taking another look. International journal of corporate social responsibility, 1(1), 3. https://doi.org/10.1186/s40991-016-0004-6

Coppa, M., y Sriramesh, K. (2013). Corporate social responsibility among SMEs in Italy. Public Relations Review, 39(1), 30-39. https://doi.org/10.1016/j.pubrev.2012.09.009

Coronado P., F.H. (2016). Objetivos de Desarrollo Sostenible. Revista Universidad de La Salle, (70), 7-11.

Crespo R., F. (2010). Entre el concepto y la práctica: responsabilidad social empresarial. Estudios Gerenciales, 26(117), 119-130.

D’Andreis Z., A. C. (2013). Responsabilidad social empresarial RSE: un estudio desde sus teorías, Precursores y críticos. Adgnosis, 2(2), 49-63

Dávila, C. (2001). Teorías organizacionales y administración. McGraw Hill.

Dentchev, N. A. (2004). Corporate Social Performance as a Business Strategy. Business Ethics, 55(4), $397-412$.

Donaldson, T. y Dunfee, T.W. (1999). When ethics travel: The promise and peril of global business ethics. California Management Review, 41(4), 45-63.

Drucker, P. F. (1984). Converting social problems into business opportunities: The new meaning of corporate social responsibility. California Management Review, 26(2), 53-63.

Dubois, A. (2006). La dimensión normativa del desarrollo en la globalización: una visión crítica de los Objetivos del Milenio. Revista de Dirección y Administración de Empresas. 13, 32-52.

Enderle, G. (2004). Global competition and corporate responsibilities of small and medium- sized Enterprises. Business Ethics: A European Review, 13(1), 51-63.

Fraj-Andrés, E., López-Pérez, M. E., Melero-Polo, I., y Vázquez-Carrasco, R. (2012). Company image and corporate social responsibility: reflecting with SMEs' managers. Marketing Intelligence \& Planning, 30(2), 266-280.

Franco A., M., y Urbano P., D. (2010). El éxito de las Pymes en Colombia: Un Estudio de Casos en El Sector Salud. Estudios Gerenciales, 26(114), 77-97.

Freeman, R. E. (1984). Strategic management: A stakeholder approach. Pitmat, London.

Friedman, M. (1970). The Social Responsibility of Business is to Increase its Profits. The New York Times Magazine. 
García de los Salmones, M.d.M., Herrero C., A., \& Rodríguez d. B., I. (2005). Influence of corporate social responsibility on loyalty and valuation of services. Journal of business ethics, 61(4), 369-385.

Hamburger, A. A. (2007). Ética y Responsabilidad Social de las Organizaciones. Revista Electronica Management, 18(28), 109-124.

Herrera M., J., Larrán J., M., Martínez C., I., y Martínez-Martínez, D. (2016). Relationship between corporate social responsibility and competitive performance in Spanish SMEs: Empirical evidence from a stakeholders' perspective. BRQ Business Research Quarterly, 19(1), 55-72.

Herrera M., J., Larrán J., M., y Martínez-Martínez, D. (2013). Relación entre responsabilidad social y performance en las pequeñas y medianas empresas: Revisión bibliográfica. Cuadernos de Gestion, 13(2), 39-65.

Herrera M., J., Larrán J., M., y Martínez-Martínez, D. (2013). Relación entre responsabilidad social y performance en las pequeñas y medianas empresas: Revisión bibliográfica. Cuadernos de Gestion, 13(2), 39-65.

Herrera M., J., Larrán M., J., Lechuga S, M. P. y Martínez-Martínez, D. (2015b). Responsabilidad social en las pymes: análisis exploratorio de factores explicativos. Revista de Contabilidad, 19(1), 31-44.

Herrera M., J., Larrán M., J., Lechuga S, M. P. y Martínez-Martínez, D. (2015b). Responsabilidad social en las pymes: análisis exploratorio de factores explicativos. Revista de Contabilidad, 19(1), 31-44.

Herrera M., J., Larrán M., J., Lechuga S., M. P., y Martínez-Martínez, D. (2015a). Evolución de la literatura sobre la responsabilidad social en pymes como disciplina científica. Revista Europea de Dirección y Economía de la Empresa, 24(2), 117-128.

Herrera M., J., Larrán M., J., Lechuga S., M. P., y Martínez-Martínez, D. (2015a). Evolución de la literatura sobre la responsabilidad social en pymes como disciplina científica. Revista Europea de Dirección y Economía de la Empresa, 24(2), 117-128.

Ibrahim, N. A., \& Angelidis J. A. (1993) Corporate Social Responsibility: A Comparative Analysis of Perceptions of Top Executives and Business Students. The Mid-Atlantic Journal of Business 29(3): 303-314

Laudal, T. (2011). Drivers and barriers of CSR and the size and internationalization of firms. Social Responsibility Journal, 7(2), 234-256.

Looser, S., y Wehrmeyer, W. (2016). Ethics of the firm, for the firm or in the firm? purpose of extrinsic and intrinsic CSR in switzerland. Social Responsibility Journal, 12(3), 545-570.

Maignan, I. y Ferrell, O. C. (2003). Nature of corporate responsibilities: perspectives from American, French, and German consumers. Journal of Business Research, 56(1), 55-67.

Maignan, I., \& Ferrell, O. C. (2000). Measuring Corporate Citizenship in Two Countries: The Case of the United States and France. Journal of Business Ethics, 23(3), 283-297. DOI: 10.1023/a:1006262325211

Malaquias, R. F., Malaquias, F. F., \& Hwang, Y. (2016). Effects of information technology on corporate social responsibility: Empirical evidence from an emerging economy. Computers in Human Behavior, 59, 195201.

Mellado, C. (2009). Responsabilidad Social Empresarial en las Pequeñas y Medianas Empresas Latinoamericanas. Revista de Ciencias Sociales (RCS), XV(1), 24-33. 
Miranda, D. D. P., y Cantallops, A. S. (2013). La práctica de la responsabilidad social empresarial. Estudio de caso en el sector turístico. Innovar, 23(49), 101-114.

Nieto A., M., y Fernández G., R. (2004). Responsabilidad social corporativa: la última innovación en management. Universia, Business Review, 1(1), 28-39

Ocampo-López, O. L., García-Cortés, J. A., Ciro-Ríos, L. S., y Forero-Páez, Y. (2015). Responsabilidad social en pequeñas empresas del sector de Alimentos y Bebidas de Caldas. Entramado, 11(2), 72-90.

Özmen, Ö. (2010). Business ethics research with an accounting focus: a bibliometric analysis from 1988 to 2007. Journal of Business Ethics, 93(1), 137-160.

Park, B. I., y Ghauri, P. N. (2015). Determinants influencing CSR practices in small and medium sized MNE subsidiaries: A stakeholder perspective. Journal of World Business, 50(1), 192-204.

Pérez R., A., García de los Salmones S., M. D. M., \& Rodríguez del Bosque R., I. A. (2008). Las dimensiones de la responsabilidad social de las empresas como determinantes de las intenciones de comportamiento del consumidor. Revista Asturiana de Economía (41), 127-147

Pinkston, T. S., \& Carroll, A. B. (1996). A retrospective examination of CSR orientations: Have they changed? Journal of Business Ethics, 15(2), 199-206. DOI: 10.1007/bf00705587

Popa, O. (2010). Organizational Social Responsibility-State of The Art. Revista tinerilor economişti, 59-68.

Preuss, L., \& Perschke, J. (2010). Slipstreaming the larger boats: Social responsibility in medium-sized businesses. Journal of Business Ethics, 92(4), 531-551.

Rahim, M. M., y Wisuttisak, P. (2013). Corporate social responsibility-oriented compliances and SMEs access to global market: Evidence from Bangladesh. Journal of Asia-Pacific Business, 14(1), 58-83.

Sen, S., y Cowley, J. (2013). The relevance of stakeholder theory and social capital theory in the context of CSR in SMEs: An Australian perspective. Journal of Business Ethics, 118(2), 413-427.

Tang, Z., y Tang, J. (2015). The influence of stakeholder-firm power difference on corporate social responsibility of chinese small and medium-sized enterprises. World Review of Entrepreneurship, Management and Sustainable Development, 11(4), 414-428.

Vargas, A. (2002). Modelo para la elaboración de un plan de negocios para las empresas pequeñas y medianas. Estudios Gerenciales, (82), 93-110.

Vera A., L. D., \& Peláez V., D. C. (2013). Análisis de los dominios ético, legal y económico de la responsabilidad social empresarial: un caso empresarial. Civilizar. Ciencias Sociales y Humanas, 13(25), 85-101.

Vitell, S.J. y Paolillo, J.G. (2004). A cross-cultural study of the antecedents of the perceived role of ethics and social responsibility. Business Ethics, 13(2-3), 185-199.

Vives, A., Corral, A., y Isusi, I. (2005). Responsabilidad social de la empresa en las PYMEs de Latinoamerica. Washington: BID.

Wang, H., Tong, L., Takeuchi, R., \& George, G. (2016). Corporate social responsibility: An overview and new research directions. Academy of management journal, 59(2), 534-544.

Esta obra está bajo una Licencia Creative Commons

Attribución-NoCommercial 4.0 International

(cc) $\mathbf{B Y}-\mathrm{NC}$ 